\title{
Can manipulation under anesthesia alone provide clinical outcomes similar to arthroscopic circumferential capsular release in primary frozen shoulder (FS)?: the necessity of arthroscopic capsular release in primary FS
}

\author{
Seung-Jin Lee, Jun-Hyuk Jang, Yoon-Suk Hyun \\ Department of Orthopedic Surgery, Kangdong Sacred Heart Hospital, Seoul, Korea
}

\begin{abstract}
Background: We evaluated the need for arthroscopic capsular release (ACR) in refractory primary frozen shoulder (FS) by comparing clinical outcomes of patients treated with ACR and manipulation under anesthesia (MUA).

Methods: We assessed patients with refractory primary FS, 57 patients (group A) who were treated with MUA and 22 patients (group B) who were treated with ACR. In group A, manipulation including a backside arm-curl maneuver was performed under interscalene brachial block. In group B, manipulation was performed only to release the inferior capsule before arthroscopic circumferential capsular release, which was carried out for the unreleased capsule after manipulation. Pain, range of shoulder motion, and American Shoulder and Elbow Surgeons score were recorded at 1 week, 3 months, 6 months, and 1 year after surgery. We compared outcome variables between treatment groups and between diabetics and non-diabetics and also evaluated the numbers of patients receiving additional intra-articular steroid injection.

Results: Outcome variables at 3 months after surgery and improvements in outcome variables did not differ between groups. Group A showed significantly better results than group B in the evaluation of pain and range of motion at 1 week. Diabetics showed comparable outcomes to non-diabetics for most variables. Eleven patients required additional steroid injections between 8 to 16 weeks after surgery: $12.2 \%$ in group A, 18.2\% in group B. Additional injections were given three times more often in diabetics compared to non-diabetics.

Conclusions: MUA alone can yield similar clinical outcomes to ACR in refractory FS.
\end{abstract}

Keywords: Frozen shoulder; Manipulation; Capsular release; Diabetes

\section{INTRODUCTION}

Even if the natural course of primary frozen shoulder (FS) is mostly self-limiting, some patients experience prolonged disability with considerable pain and disability that affect activities of daily life $[1,2]$. Moreover, some patients fail to achieve desired outcomes with non-operative management. Given the natural history of adhesive capsulitis and the high proportion of patients who do well with nonsurgical management, a trial of at least 6 months of nonsurgical management is normally recommended

Received: September 30, 2020 Revised: November 1, 2020 Accepted: November 4, 2020

Correspondence to: Yoon-Suk Hyun

Department of Orthopedic Surgery, Kangdong Sacred Heart Hospital, 150 Seongan-ro, Gangdong-gu, Seoul 05355, Korea

Tel: +82-2-2224-2230, Fax: +82-2-489-4391, E-mail: yshyun72@gmail.com, ORCID: https://orcid.org/0000-0003-3826-2527

Financial support: None.

Conflict of interest: None.

Copyright@ 2020 Korean Shoulder and Elbow Society. All Rights Reserved.

This is an Open Access article distributed under the terms of the Creative Commons Attribution Non-Commercial License (http://creativecommons.org/licenses/by-nc/4.0/) which permits unrestricted non-commercial use, distribution, and reproduction in any medium, provided the original work is properly cited. 
before considering surgical management options: manipulation under anesthesia (MUA), arthroscopic capsular release (ACR), or the combination of both [3].

Although ACR is gaining in popularity with recent advances in arthroscopic technique and has shown promising results comparable to those of other treatment modalities [4-6], MUA is a traditionally well-established treatment for FS that is nevertheless controversial due to potential complications (e.g., proximal humerus fractures, shoulder dislocation, brachial plexus stretching injury, rotator cuff injury, and recurrent stiffness) [7-10]. There are no good quality randomized controlled trials in favor of ACR in comparison to MUA [11]; in two previous studies, the superior treatment was not identified $[11,12]$ and manipulation was performed under general anesthesia. Manipulation for FS is usually performed under general anesthesia, but is also performed under interscalene brachial plexus block (ISB) anesthesia and obtains favorable outcomes [13-16].

No previous studies have compared the clinical outcomes of manipulation under ISB anesthesia and ACR. We evaluated differences in clinical outcomes between manipulation under ISB and ACR in refractory primary FS to determine whether ACR is necessary if manipulation under interscalene brachial plexus anesthesia is performed following our novel method. We hypothesized that MUA alone would provide similar clinical outcomes as ACR in primary FS.

\section{METHODS}

The protocol of this study was reviewed and approved by Institutional Review Board of Kangdong Sacred Heart Hospital (IRB No. 2019-09-016). Written informed consents were obtained.

\section{Study Design and Participants}

We performed a retrospective analysis of a prospectively collected, single surgeon (YSH), single institution, consecutive series of patients with FS. From March 2015 to Mar 2018, 79 patients who were diagnosed with primary FS in our hospital were treated with MUA or ACR. The definition of FS in this study followed the American Shoulder and Elbow Surgeons (ASES) consensus study by Zuckerman and Rokito [17] and was characterized by functional restriction of both active and passive shoulder motion for which radiographs of the glenohumeral joint were essentially unremarkable except for the possible presence of osteopenia. In all patients, shoulder magnetic resonance imaging was used to screen for the coexistence of any other shoulder lesions before indicated management was performed. Patients with rotator cuff tear, shoulder osteoarthritis, calcified tendinitis, hemiplegia after stroke, bone metastasis in the shoulder region, history of shoulder fractures, and history of shoulder surgeries were excluded. Diabetic FS patients were not excluded because while diabetes is a possible predisposing factor based on statistical data, it is not known to be a cause of FS [18]. Refractory FS was defined as follows: refractory to conservative treatment (intra-articular steroid injections and physical therapy) for at least 6 months and documented restriction of both passive and active glenohumeral and scapulothoracic motion of equal to or less than $100^{\circ}$ of elevation, and less than $50 \%$ of external rotation, as compared to the contralateral side [11].

Both treatment modalities were indicated by refractory FS. There were no differences in indications between the two modalities. ACR after MUA was used from January 2015 to May 2017, and the two modalities were used randomly without special indications for either during the 5 months from January 2017 onward. After this period of overlap, we recognized that the two modalities showed similar clinical outcomes. MUA alone was used after May 2017 to facilitate comparisons of the modalities. All 79 patients treated during this period who were followed closely over 6 months after treatments were reviewed. A total of 57 patients (group A) were treated with MUA and 22 patients (group B) were treated with ACR after manipulation. We evaluated range of motion (ROM; passive forward elevation, external rotation arm at side, and thumb reach along the vertebral spine, in which the thumb points up), visual analog pain score, and ASES score preoperatively and at 1 week, 3 months, 6 months, and 1 year after surgery. All outcome variables in this study were measured by trained residents and physician assistants under the supervision of the senior author (YSH). We consider the number of vertebral spines in which the patient's thumb can reach up, which we refer to as thumb-to-spine, to be better than degree of internal rotation for the evaluation of surgical outcomes. When the patient's thumb reaches up over the thoracolumbar junction, we defined it as a pass. Regarding the evaluation of pain severity, we asked the patient for an average value, taking into account pain while sleeping at night, pain while resting, and pain during everyday activities. We compared results for the two groups and analyzed them statistically. We compared outcome measurements, pretreatment period, follow-up period, and number of additional intra-articular steroid injections between groups. We also compared all variables between diabetic and non-diabetic patients.

\section{Study Procedures}

In group A, all MUA procedures were performed by a single surgeon (YSH) following a standardized, identical protocol. MUA 
was performed in outpatient settings. The patient was made to lie down in a supine position after interscalene plexus block was performed by the anesthesiologist. Before MUA, intra-articular steroid injections were performed to control post-MUA inflammation and pain (triamcinolone $40 \mathrm{mg}, 1 \mathrm{~mL}+$ lidocaine $0.1 \%, 5$ $\mathrm{mL}+$ sterile normal saline, $4 \mathrm{~mL}$ ). Manipulations were first performed at forward flexion with scapular stabilization by an assistant, and then at external rotation with the arm at the side, and finally at $90^{\circ}$ abduction (Fig. 1) [16]. Next, internal rotation was performed with the arm at $90^{\circ}$ abduction, while the assistant pushed the shoulder girdle downward to the floor (Fig. 1A) [16]. During $90^{\circ}$ abduction with full internal rotation, further abduction can lead to further capsular release indicated by an audible sound (Fig. 1B). Next, horizontal adduction with scapular stabilization was performed. After the patient was made to sit, we placed the patient's hand behind their back and then pushed the patient's arm backwards, while pushing the patient's hand up with forced adduction (Fig. 2). This last step is the "backside arm-curl maneuver." Most of the time, a recognizable tearing sound was heard during each step. The sequence was repeated until the maximum ROM was acquired. A short lever arm with the elbow flexed at $90^{\circ}$ is used to prevent fractures and brachial plexus traction injuries. After MUA completion, the recovered range of shoulder motion was confirmed with the patient (Fig. 3). The patient was instructed to start passive exercise programs immediately after MUA to maintain the restored ROM, in which forward elevation and the backside arm-curl maneuver were emphasized (Fig. 4). The patient was also instructed to perform a self-assisted stretch for 5 minutes every hour until the next visit. Patients were allowed to go home after recovery from ISB anesthesia.

In group B, manipulation preceded arthroscopic procedures and was performed at forward elevation only for the safe release
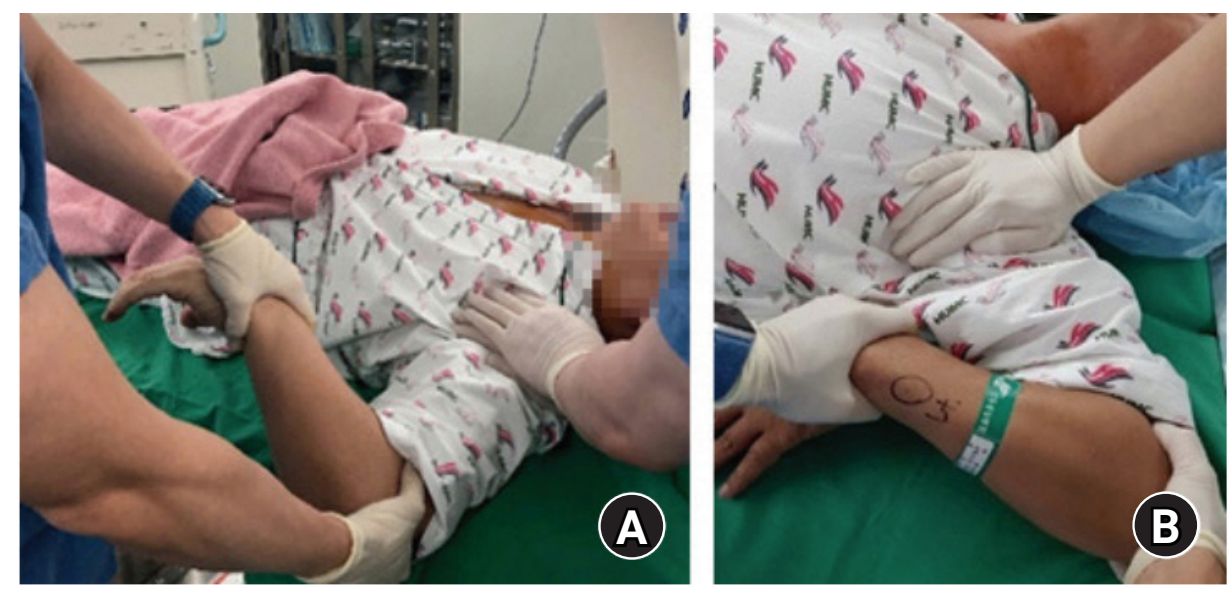

Fig. 1. (A) Internal rotation with the arm at $90^{\circ}$ abduction, with the assistant pushing the shoulder girdle downward toward the floor was performed to complete the posterior and inferior capsule tear. (B) With $90^{\circ}$ abduction with full internal rotation, further abduction can result in further capsular release with an audible sound.
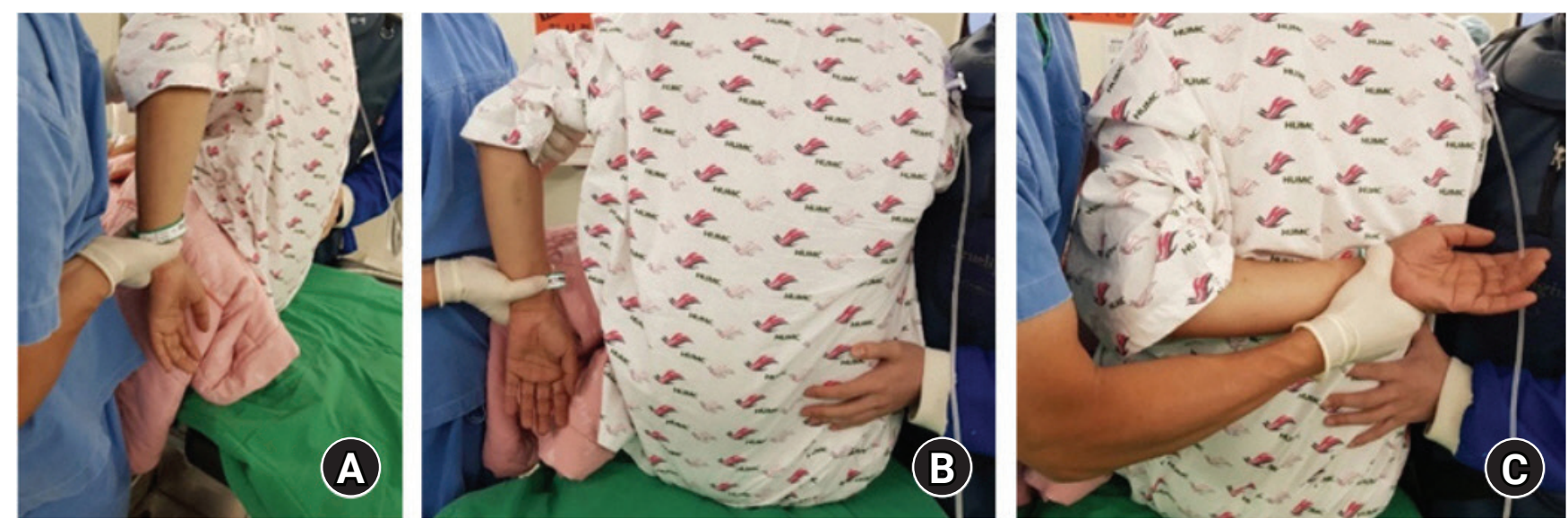

Fig. 2. Backside arm-curl maneuver. (A) It is often hard to place the patient's hand on the midline of the back in the sitting position even after full restoration of internal rotation in the supine position. $(B, C)$ After the patient was made to sit, we placed the patient's hand behind their back and then pushed the patient's arm backwards, while pushing the patient's hand up with forced arm adduction. 
of the inferior capsular area after interscalene plexus block and general anesthesia. Therefore, the backside arm-curl maneuver in the sitting position was omitted in group B. During ACR, the unreleased inferior capsule, anterior capsule, posterior capsule, and anterior part of the superior capsule (superior glenohumeral ligament and coracohumeral ligament) were released. After arthroscopic $360^{\circ}$ circumferential capsular release, an epidural catheter was inserted into the glenohumeral joint space for intra-articular steroid injection. The day after surgery, the passive exercise program was started after intra-articular steroid injection. The exercise program in group B also emphasized forward elevation and the backside arm curl maneuver. The patients remained in hospital until they showed more than $135^{\circ}$ of forward elevation and could reach higher than the fifth lumbar vertebra during the backside arm curl maneuver at least once. The length of hospital stay after surgery averaged 1 or 2 days. We informed the patient that the sooner they began exercise the better regarding the result of ROM restoration. The patient was also instructed to perform a self-assisted stretch for 5 minutes every hour until the next visit. All patients received instructions to perform re-
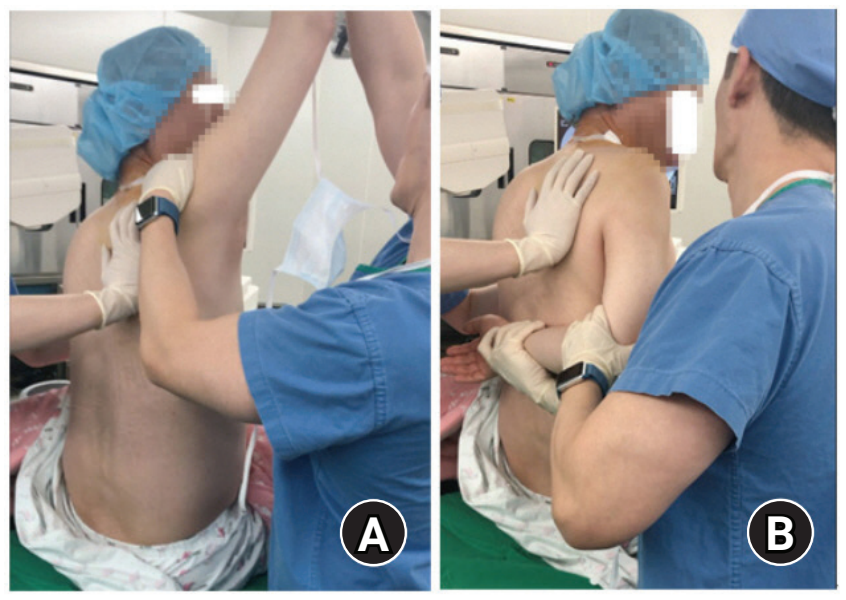

Fig. 3. (A, B) After manipulation under anesthesia completion, range of motion was confirmed with the patient. habilitation on their own without help.

Within 1 week after discharge, all patients in both groups visited the outpatient clinic to check whether they had maintained the restored ROM in group A and how much ROM had been restored in group B. All patients were evaluated according to our follow-up schedule. In group B, additional visits during the month after surgery were performed to encourage patients to perform rehabilitation exercises because most group B patients did not show significant improvements in pain and ROM at the first visit. During the follow up period, we performed intra-articular steroid injections (triamcinolone $40 \mathrm{mg}, 1 \mathrm{~mL}$ +lidocaine $0.1 \%, 5 \mathrm{~mL}+$ sterile normal saline, $4 \mathrm{~mL}$ ) if the patient complained of aggravated pain impeding ROM exercises and night sleep.

For statistical analysis, independent t-tests (age), Fisher's exact tests (sex) and Mann-Whitney U-tests (ASES scores, ROM, pretreatment periods, follow-up periods, pain VAS) were used with significance set at the 5\% level (IBM SPSS 22.0; IBM Corp., Armonk, NY, USA).

\section{RESULTS}

There were no significant differences in demographic data between groups (Table 1). Twenty-four patients had diabetes mellitus $(30.4 \%, 24$ of 79 patients). Three patients had thyroid disease

Table 1. Demographic data for both study groups

\begin{tabular}{lccc}
\hline Variable & Group A & Group B & p-value \\
\hline Number of patients & 57 & 22 & \\
Age (yr) & $55.3 \pm 8.5$ & $53.9 \pm 6.4$ & 0.474 \\
Male:female & $24: 33$ & $8: 14$ & \\
Diabetes & $17(29)$ & $7(32)$ & 0.443 \\
Thyroid disease case & 3 & 0 & \\
Pretreatment period (mo) & $6.4 \pm 3.7$ & $6.6 \pm 4.1$ & 0.391 \\
Follow-up period (mo) & $7.68 \pm 1.7$ & $7.22 \pm 1.6$ & 0.285 \\
\hline
\end{tabular}

Values are presented as mean \pm standard deviation or number (\%).
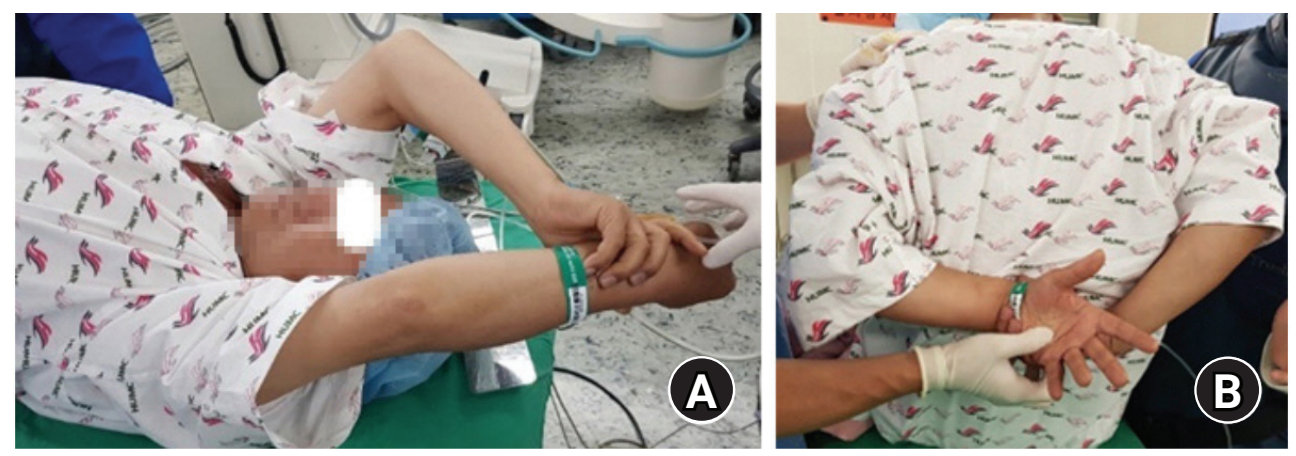

Fig. 4. (A, B) The patient was instructed to initiate passive exercise programs right after manipulation under anesthesia to maintain the restored range of motion, in which forward elevation and the backside arm-curl maneuver were emphasized. 
in group A, while none did in group B. Preoperative outcome variables, outcome variables at 3 months after surgery, and amount of improvement in all variables (ASES score, pain VAS, and ROM) did not differ between groups (Tables 1 and 2). However, at the last visit, the degree of forward elevation was better in group A than group $B(p=0.029)$ although other outcome variables did not differ between groups.

No patients were able to raise their thumbs higher than the thoracolumbar junction. At the first visit after surgery, all patients in group A were graded as passing. In later evaluations, all patients in group A continued to be graded as passing. However, in the ACR group, only five patients passed at their first visit. The numbers of patients who passed were 12 at 1 month, 21 at 3 months, and 22 (all patients) at the last visit. Group A showed significantly better results than group B for pain and ROM (Table 1).

No patients in either group (manipulation under ISB only and ACR with MUA) showed decreased ROM during the follow-up period. Seven patients among 57 treated by MUA (12.2\%) and four by ACR (18.2\%) complained of aggravated pain between 8 and 16 weeks (median value, 10 weeks) after surgery. The pain in these 11 patients subsided after a single intra-articular steroid injection and did not recur during the follow-up period. These 11 patients did not show any significant differences in ROM, ASES score, or severity of pain at final evaluation. No serious complications after manipulation, such as shoulder dislocation, rotator cuff tear, or brachial plexus palsy were reported in our patients.

We included 24 diabetic patients $(30.4 \%, 24 / 79)$ in this study. When outcome variables were evaluated without grouping, diabetic patients showed slightly worse outcomes at 3 months after surgery, but no significant little differences in last values or improvement of outcome variables (Table 3). The results were different when each group was analyzed separately. There were no differences in clinical outcomes between diabetics and non-diabetics in group A (Table 3). In group B, diabetics had worse outcomes at 3 months after surgery and at the last visit, but there were no differences in improvement of ASES score or ROM except for pain severity (Table 3). Among patients who needed additional intra-articular steroid injections, $41.2 \%$ of diabetic patients (7/17) and $7.5 \%$ of non-diabetic patients (3/40) in group A needed additional injections, and $57.1 \%$ of diabetic patients (4/7) and $11.1 \%$ of non-diabetic patients (2/18) in group B needed additional injections.

\section{DISCUSSION}

We did not detect differences in overall outcomes between patients treated with MUA and ACR. Interestingly, pain severity

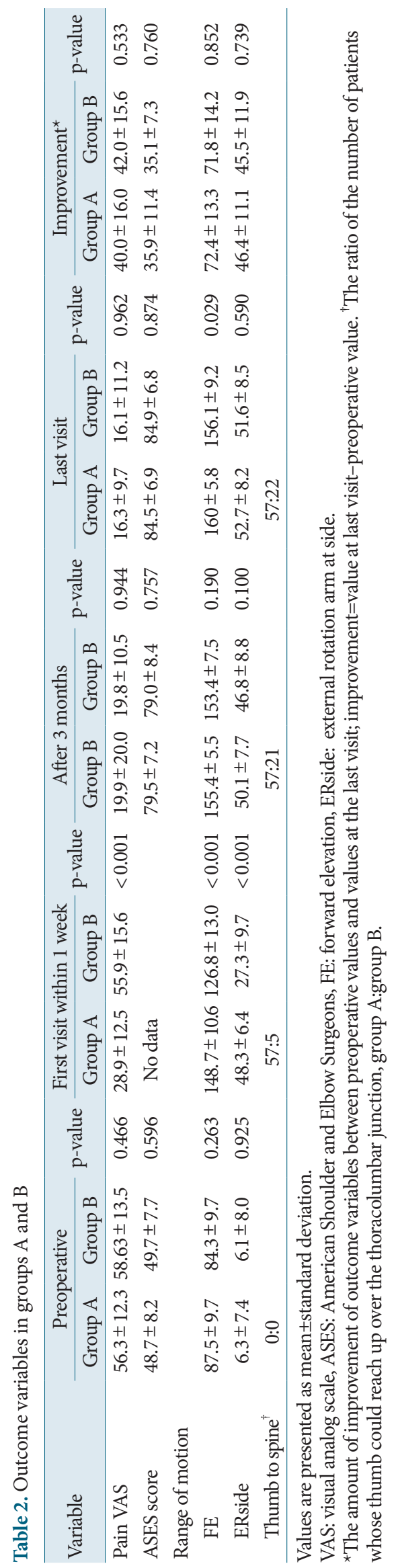




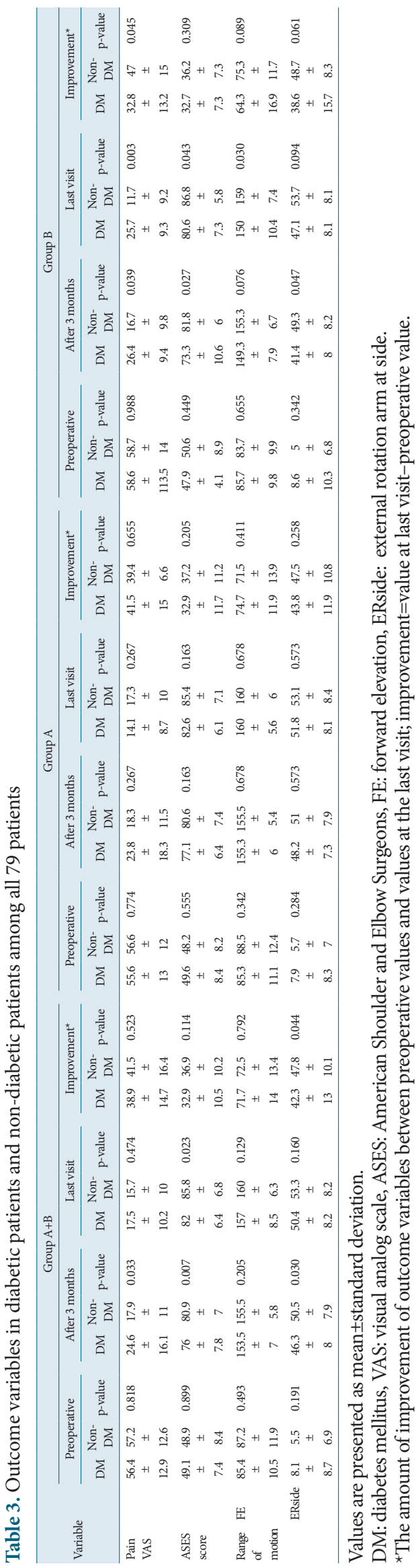

and ROM scores were better in the MUA group than the ACR group at the first visit, but there were no differences in final outcomes or improvement between groups.

Eleven patients complained of aggravated pain between 8 and 16 weeks (median, 10 weeks) after each procedure: 7 of 57 patients $(12.2 \%)$ in the MUA group and 4 of 22 patients (18.2\%) in the ACR group. Regarding the recurrence of pain, the number of diabetic patients requiring additional injections was three times higher than non-diabetic patients.

Given the natural history of adhesive capsulitis and the high proportion of patients who do well with nonsurgical management, a trial of at least 6 months of nonsurgical management is generally recommended before considering surgical management options [3]. MUA and ACR are the two most common surgical management strategies. There are no good quality randomized controlled trials with findings showing the superiority of ACR in comparison to MUA [11], but ACR is favored over MUA because it is believed to allow a more controlled and complete release of the contracted capsule, to reduce the risk of iatrogenic injury compared to MUA, and also resulted in favorable shortterm and long-term outcomes [11,19-24]. In our study, patients treated with MUA showed similar outcomes to ACR and did not report any serious iatrogenic complications.

There is controversy regarding the optimal method of ACR. Some authors have recommended $360^{\circ}$ capsular release to restore normal ROM, whereas others have determined that partial capsular release (anterior-inferior capsule) is sufficient [4,22,24,25]. In group B, we performed $360^{\circ}$ circumferential capsular release, in which the coracohumeral ligament and superior glenohumeral ligament as well as the anterior-inferior-posterior capsule were released, because we believe that release of the coracohumeral ligament and superior glenohumeral ligament is important $[4,26$ 28]. In three patients who were not included in this study, we performed arthroscopic examinations to determine the extent of capsular release after MUA that included the backside arm-curl maneuver. We found that the anterior middle glenohumeral ligament, inferior glenohumeral ligament (anterior and posterior band), and posterior capsule below the equator were successfully released. Previous studies supporting the efficacy of arthroscopic anteroinferior release and our clinical findings that MUA with backside arm-curl could restore nearly normal ROM, constitute evidence that ACR is unnecessary.

Recent developments in ultrasound technology have enabled its use with brachial plexus anesthesia or cervical root block [1316,29]. Previous reports described good results like those of the present study in primary FS patients with or without diabetes. However, no previous studies have compared outcomes of ma- 
nipulation under ISB and ACR in patients with refractory primary FS. Our study may be the first to compare the outcomes of patients treated by both modalities in a single institution. Our novel manipulation techniques resulted in comparable clinical outcomes to ACR.

Only two previous studies compared outcomes of MUA and ACR in patients with refractory FS $[11,12]$. Neither determined which of the two treatment modalities is superior. Grant et al. [11] conducted a systematic review and concluded that given the low level of evidence and lack of direct comparisons, there are no clear differences in shoulder ROM or patient-reported outcomes when comparing MUA to ACR for the treatment of refractory primary FS. Kim et al. also reported similar results, that MUA (general anesthesia) offered equivalent clinical outcomes compared with ACR $\left(360^{\circ}\right.$ circumferential release) in the early period after the procedure [12]. However, they recommended that clinicians should consider MUA as an option before choosing ACR in patients with refractory FS because MUA is simple, safe, and cost-effective.

In this study, we introduced and described the novel backside arm-curl maneuver in the sitting position. In previous studies of MUA in primary FS, the descriptions of manipulation procedures are brief or variable, and procedures are generally not conducted in the sitting position $[12,15,18,20,25]$. For the successful restoration of ROM in MUA, the restoration of the height of thumb reach up the spine as well as forward elevation is very important. We found that sufficient shoulder extension, backward elevation, and adduction in the sitting position are critical for the restoration of the height of the thumb reaching up the spine to normal range and tearing sounds are audible during this procedure. Performance of the backside arm-curl maneuver in the sitting position is extremely important.

Woods and Loganathan [30] found that patients with successful outcomes have significant improvement in pain within three to four days after MUA, and improvement in ROM within about three weeks. Kraal et al. [29] also found that an initial period of one to 2 weeks of intensive physiotherapy after MUA is essential to prevent recurrence of restrictions and advocated more aggressive rehabilitation with intensive stretching and ROM exercises in the first weeks after MUA to preserve the obtained ROM. The importance of early rehabilitation is the same in ACR and MUA in terms of surgical treatment for joint stiffness [20]. We also emphasized the early restoration of ROM in both groups. Patients in the MUA-only group showed earlier improvements in pain and ROM at first visit than the ACR group, although there was no significant difference at 3 months between groups. Final outcomes are important, but it is also important to treat pain quickly and restore ROM. The faster recovery of ROM and pain, the better the patient's treatment compliance.

When using our method of performing MUA, we were able to immediately confirm ROM recovery during manipulation under ISB. ISB and intra-articular steroid injections minimized pain during the rehabilitation exercise, which was carried out immediately after MUA, so ROM restored with the procedure was well maintained. Immediate initiation of exercise after manipulation for 6 to 8 hours becomes possible thanks to the use of ISB, and intra-articular steroid injections also reduce pain on exercise after ISB wears off. Less invasive procedures than ACR are also helpful to reduce pain on rehabilitation. Patients in the ACR group reported more pain during joint exercise compared to MUA at their first outpatient visits.

There are clear differences difference between presence and absence of restored ROM. In manipulation under ISB, there is no $\mathrm{ROM}$ to restore, and full restoration is accomplished as soon as manipulation is complete. In MUA or ACR under general anesthesia, the immediate confirmation of restoration of ROM with the patient is difficult or impossible, and some patients doubt the success of manipulation if rehabilitation is onerous or they do not restore normal ROM.

Regarding the recurrence of FS after MUA, two previous studies of MUA reported rates of recurrence of between $10 \%$ and $40 \%$ [30,31]. Woods and Loganathan [30] performed further MUA if there were no improvements in ROM or pain 3 months after MUA. They also reported a period of recurrence in some patients (17.8\%, 141 of 792 shoulders) between 6 and 8 weeks after treatment, which could be accounted for by the loss of the effect of the intra-articular steroid injection. In our study, no patients experienced decreased ROM during the follow-up period, while 11 patients complained of aggravated pain between 8 and 16 weeks after the procedure: seven of 57 patients (12.2\%) in the MUA group and four of 22 patients (18.2\%) in the ACR group.

FS is strongly associated with diabetes and is two to four times more common in diabetic patients than in the general population [32-36]. FS in patients with diabetes tends to have a more severe and protracted course, and to be difficult to treat $[32,37,38]$. Some researchers have suggested that patients with diabetes should consider ACR, but most of our patients experienced good outcomes after manipulation alone [17]. However, in our study, diabetic patients showed slightly worse outcomes compared with non-diabetic patients although outcome variables in the ACR group were not significantly different. We observed no differences in any outcome variables in the MUA group, a finding that is consistent with those of other reports and suggests that diabetes is not a contraindication for MUA [16,18,31,34]. Re- 
garding recurrence after surgery, Jenkins et al. reported that repeat MUA was required in $36 \%$ of diabetic patients compared with $15 \%$ of nondiabetic patients, and Woods and Loganathan [30] reported that patients with type-1 diabetes mellitus were at $38 \%$ increased risk of requiring further MUA, compared with $18 \%$ increased risk among a group that included both diabetics and non-diabetic patients [31]. Regarding recurrence pain, diabetic patients were more than three times as likely to experience recurrence than non-diabetics (41.2\% vs. $7.5 \%$ in group A and $57.1 \%$ vs. $11.1 \%$ in group B) in our study.

This study has several limitations. It is a retrospective, non-randomized study with a relatively small sample size. Sample sizes are also different between groups. There may be subtle differences in the indications of the two procedures because ACR requires general anesthesia, so it can be more difficult for patients to accept than MUA, which is performed under regional anesthesia. We developed and introduced the backside arm-curl maneuver with good success, but further research is needed to validate this procedure. Future studies should include evaluations of ROM improvement after manipulation with and without the backside arm-curl maneuver. Comparisons between manipulation under ISB and general anesthesia should also be performed in the future. Despite these limitations, our study is the first to compare the clinical results of manipulation under ISB with ACR.

In primary FS, manipulation under ISB alone can result in similar and favorable clinical outcomes with ACR in refractory cases. Regardless of type of surgery, clinical outcomes in patients with diabetes were similar to those without diabetes. However, diabetic patients often require additional intra-articular steroid injections.

\section{ORCID}

Seung-Jin Lee

Jun-Hyuk Jang

Yoon-Suk Hyun https://orcid.org/0000-0001-8951-0039

https://orcid.org/0000-0002-9640-1700

https://orcid.org/0000-0003-3826-2527

\section{REFERENCES}

1. Reeves B. The natural history of the frozen shoulder syndrome. Scand J Rheumatol 1975;4:193-6.

2. Shaffer B, Tibone JE, Kerlan RK. Frozen shoulder: a long-term follow-up. J Bone Joint Surg Am 1992;74:738-46.

3. Hsu JE, Anakwenze OA, Warrender WJ, Abboud JA. Current review of adhesive capsulitis. J Shoulder Elbow Surg 2011; 20:502-14.
4. Cvetanovich GL, Leroux TS, Bernardoni ED, et al. Clinical outcomes of arthroscopic $360^{\circ}$ capsular release for idiopathic adhesive capsulitis in the lateral decubitus position. Arthroscopy 2018;34:764-70.

5. Gallacher S, Beazley JC, Evans J, et al. A randomized controlled trial of arthroscopic capsular release versus hydrodilatation in the treatment of primary frozen shoulder. J Shoulder Elbow Surg 2018;27:1401-6.

6. De Carli A, Vadalà A, Perugia D, et al. Shoulder adhesive capsulitis: manipulation and arthroscopic arthrolysis or intra-articular steroid injections. Int Orthop 2012;36:101-6.

7. Loew M, Heichel TO, Lehner B. Intraarticular lesions in primary frozen shoulder after manipulation under general anesthesia. J Shoulder Elbow Surg 2005;14:16-21.

8. Amir-Us-Saqlain H, Zubairi A, Taufiq I. Functional outcome of frozen shoulder after manipulation under anaesthesia. J Pak Med Assoc 2007;57:181-5.

9. Milch H. Brachial palsy after manipulation of frozen shoulder. N Engl J Med 1954;250:429-30.

10. Uppal HS, Evans JP, Smith C. Frozen shoulder: a systematic review of therapeutic options. World J Orthop 2015;6:263-8.

11. Grant JA, Schroeder N, Miller BS, Carpenter JE. Comparison of manipulation and arthroscopic capsular release for adhesive capsulitis: a systematic review. J Shoulder Elbow Surg 2013; 22:1135-45.

12. Kim DH, Song KS, Min BW, Bae KC, Lim YJ, Cho CH. Early clinical outcomes of manipulation under anesthesia for refractory adhesive capsulitis: comparison with arthroscopic capsular release. Clin Orthop Surg 2020;12:217-23.

13. Ando A, Hamada J, Hagiwara Y, Sekiguchi T, Koide M, Itoi E. Short-term clinical results of manipulation under ultrasound-guided brachial plexus block in patients with idiopathic frozen shoulder and diabetic secondary frozen shoulder. Open Orthop J 2018;12:99-104.

14. Saito T, Sasanuma H, Iijima Y, et al. Short-term clinical results of frozen shoulder treated with shoulder manipulation under ultrasound-guided cervical nerve root block at outpatient setting: a case series. J Orthop Sci 2017;22:275-80.

15. Kraal T, Van der Meer O, Van den Borne M, Koenraadt K, Eygendaal D, Boer R. Manipulation under anesthesia for frozen shoulders : a retrospective cohort study. Acta Orthop Belg 2019; $85: 400-5$.

16. Takahashi R, Kajita Y, Harada Y, Iwahori Y, Deie M. Clinical results of shoulder manipulation under ultrasound-guided cervical nerve root block for frozen shoulder in patients with diabetes. J Orthop 2020;21:297-301.

17. Zuckerman JD, Rokito A. Frozen shoulder: a consensus defini- 
tion. J Shoulder Elbow Surg 2011;20:322-5.

18. Wang JP, Huang TF, Ma HL, Hung SC, Chen TH, Liu CL. Manipulation under anaesthesia for frozen shoulder in patients with and without non-insulin dependent diabetes mellitus. Int Orthop 2010;34:1227-32.

19. Neviaser AS, Hannafin JA. Adhesive capsulitis: a review of current treatment. Am J Sports Med 2010;38:2346-56.

20. Neviaser AS, Neviaser RJ. Adhesive capsulitis of the shoulder. J Am Acad Orthop Surg 2011;19:536-42.

21. Farrell CM, Sperling JW, Cofield RH. Manipulation for frozen shoulder: long-term results. J Shoulder Elbow Surg 2005;14:4804.

22. Le Lievre HM, Murrell GA. Long-term outcomes after arthroscopic capsular release for idiopathic adhesive capsulitis. J Bone Joint Surg Am 2012;94:1208-16.

23. Barnes CP, Lam PH, Murrell GA. Short-term outcomes after arthroscopic capsular release for adhesive capsulitis. J Shoulder Elbow Surg 2016;25:e256-64.

24. Ranalletta M, Rossi LA, Zaidenberg EE, et al. Midterm outcomes after arthroscopic anteroinferior capsular release for the treatment of idiophatic adhesive capsulitis. Arthroscopy 2017; 33:503-8.

25. Kim YS, Lee HJ. Essential surgical technique for arthroscopic capsular release in the treatment of shoulder stiffness. JBJS Essent Surg Tech 2015;5:e14.

26. Kim YS, Chung SW, Kim JY, Ok JH, Park I, Oh JH. Is early passive motion exercise necessary after arthroscopic rotator cuff repair. Am J Sports Med 2012;40:815-21.

27. Ozaki J, Nakagawa Y, Sakurai G, Tamai S. Recalcitrant chronic adhesive capsulitis of the shoulder. Role of contracture of the coracohumeral ligament and rotator interval in pathogenesis and treatment. J Bone Joint Surg Am 1989;71:1511-5.

28. Pouliart N, Somers K, Eid S, Gagey O. Variations in the superior capsuloligamentous complex and description of a new liga- ment. J Shoulder Elbow Surg 2007;16:821-36.

29. Kraal T, The B, Boer R, et al. Manipulation under anesthesia versus physiotherapy treatment in stage two of a frozen shoulder: a study protocol for a randomized controlled trial. BMC Musculoskelet Disord 2017;18:412.

30. Woods DA, Loganathan K. Recurrence of frozen shoulder after manipulation under anaesthetic (MUA): the results of repeating the MUA. Bone Joint J 2017;99-B:812-7.

31. Jenkins EF, Thomas WJ, Corcoran JP, et al. The outcome of manipulation under general anesthesia for the management of frozen shoulder in patients with diabetes mellitus. J Shoulder Elbow Surg 2012;21:1492-8.

32. Wang K, Ho V, Hunter-Smith DJ, Beh PS, Smith KM, Weber AB. Risk factors in idiopathic adhesive capsulitis: a case control study. J Shoulder Elbow Surg 2013;22:e24-9.

33. Thomas SJ, McDougall C, Brown ID, et al. Prevalence of symptoms and signs of shoulder problems in people with diabetes mellitus. J Shoulder Elbow Surg 2007;16:748-51.

34. Theodorides AA, Owen JM, Sayers AE, Woods DA. Factors affecting short- and long-term outcomes of manipulation under anaesthesia in patients with adhesive capsulitis of the shoulder. Shoulder Elbow 2014;6:245-56.

35. Bridgman JF. Periarthritis of the shoulder and diabetes mellitus. Ann Rheum Dis 1972;31:69-71.

36. Arkkila PE, Kantola IM, Viikari JS, Rönnemaa T. Shoulder capsulitis in type I and II diabetic patients: association with diabetic complications and related diseases. Ann Rheum Dis 1996;55: 907-14.

37. Janda DH, Hawkins RJ. Shoulder manipulation in patients with adhesive capsulitis and diabetes mellitus: a clinical note. J Shoulder Elbow Surg 1993;2:36-8.

38. Griggs SM, Ahn A, Green A. Idiopathic adhesive capsulitis. A prospective functional outcome study of nonoperative treatment. J Bone Joint Surg Am 2000;82:1398-407. 